\title{
Construction of Adenovirus Vector Expressing Duck Sclerostin and its Induction Effect on Myogenic Proliferation and Differentiation In Vitro
}

Hehe Liu

Sichuan Agricultural University

Yanying Li

Sichuan Agricultural University

Qian Xu

Sichuan Agricultural University

Jianmei Wang

Sichuan Agricultural University

Chunchun Han

Sichuan Agricultural University

Lili Bai

Sichuan Agricultural University

Liang Li ( $\nabla$ liliang@sicau.edu.cn )

Farm Animal Genetic Resources Exploration and Innovation Key Laboratory of Sichuan Province, Sichuan Agricultural University https://orcid.org/0000-0001-9139-5025

\section{Research Article}

Keywords: Sclerostin, duck, myoblast, differentiation, Recombinant Adenovirus Vector.

Posted Date: August 9th, 2021

DOI: https://doi.org/10.21203/rs.3.rs-741750/v1

License: (c) (1) This work is licensed under a Creative Commons Attribution 4.0 International License.

Read Full License

Version of Record: A version of this preprint was published at Molecular Biology Reports on February 6th, 2022. See the published version at https://doi.org/10.1007/s11033-022-07151-4. 


\section{Abstract}

Background: bones and muscles originated together from the mesoderm during embryogenesis, and they can influence each other through mechanical stimulations and chemical signals. The sclerostin (SOST) is secreted from mature osteocytes. Here, we used a bird model to illustrate the potential roles of SOST on duck myoblasts to verify the hypothesis that SOST might play functions in coordinating the development of bones and muscles.

Methods and Results: Firstly, a recombinant adenovirus vector carrying duck SOST was constructed. Then, the adenovirus-mediated duck SOST was transfected into duck myoblasts. The results revealed by CCK-8 showed that the cell proliferation of myoblasts was inhibited after $12 \mathrm{~h}, 36 \mathrm{~h}$, and $48 \mathrm{~h}$ treatment by transfection of SOST. The labeling rates of EdU positive cells in the Ad-duSOST group were significantly lower than the Ad-NC group $(P<0.05)$. However, the flow cytometry showed that the G0/G1 phase number of cells was not significantly different. Furthermore, the immunofluorescence results showed that the formation of myotubes was inhibited. Subsequent transcriptome revealed that, under the ectopic expression of SOST, the genes related to Cytokine-cytokine receptor interaction, muscle development (regulation of action cytoskeleton, Wnt signaling pathway), and intercellular regulation were changed. Six of the top 20 DEGs were related to morphogenesis.

Conclusions: Our studies demonstrated that the SOST played critical roles in myoblasts differentiation by mediating the crosstalk among several pathways and transcription factors related to cell differentiation. Our data provided cellular evidence supporting the combined functions of SOST in coordinating bone and muscle co-development.

\section{Introduction}

As an intrinsic connection between bone and muscle during embryogenesis, vertebrates shared a common mesodermal origin. The proximity in anatomical position, between bone and muscle, is more beneficial to their communication through mechanical and biochemical signals [1]. The bone provides an attachment surface for skeletal muscles, and meantime, muscle affects the structure of bone. Simultaneously, they could affect each other by transmitting biochemical molecules [2]. In humans, bones and muscles tend to degenerate together during aging or disease conditions, as patients with osteoporosis are more likely to get symptoms of muscle atrophy. Moreover, a sign of bone loss is often accompanied by muscular dystrophy in long-term bedridden patients [3].

Karsenty suggested that the paracrine pathway is the core link between bones and muscles. As the largest endocrine organs in vertebrates, bones and muscles can secrete various cytokines and growth factors, participating in the perception and transmission of signals, such as mechanical stretch stimuli and changes in nutritional levels [4]. The secreted proteins from bones, such as osteocalcin [5], Sclerostin (SOST) [6], and BMPs [7], can enter muscle tissues and eventually affect myofibers' differentiation and protein metabolism through a paracrine pathway. 
The SOST, secreted from the mature osteocytes, can mainly affect the function of osteoblasts and osteoclasts [8]. It could competitively bind to the co-receptor LRP5 of the Wnt/ $\beta$-catenin signal pathway to inhibit osteoblast differentiation [9]. In vitro, the proliferation and differentiation of osteoblasts were inhibited by the addition of SOST to the cell growth medium; however, they stimulated the process of apoptosis[10, 11]. Additionally, the serum level of SOST can be increased and decreased with the prolonged bed-rest time and higher muscle load, respectively [12]. The differentiation of $\mathrm{C} 2 \mathrm{C} 12$ was promoted by adding the MLO-Y4 conditioned medium (CM) and then was inhibited by adding SOST [13]. These studies suggested that SOST might play dual roles in affecting the development of both bones and muscles. Our previous study demonstrated that the ossification process of the sternum in duck would affect pectoral muscle development. Moreover, in another experiment, we found that SOST was expressed differently between the calcified and un-calcified sternum of ducks. Therefore, it was hypothesized that the SOST might play combined roles in coordinating the co-development of bones and muscles, and the present study was designed to explore the potential effect of SOST on duck primary myoblasts, as well as provide evidence supporting the dual roles of SOST on both bones and muscles. The present study data might be used to illustrate the underlying mechanism of muscle and bone development-related diseases of humans.

\section{Materials And Methods}

\subsection{Construction of duck SOST recombinant adenovirus vector}

After predicting the restriction enzyme sites (http://nc2.neb.com/NEBcutter2/) of duck SOST coding domain sequence (CDS, XM_005026106), the NEBuilder (http://nebuilder.neb.com/) was used to design primers with overlapping sequences of pAdTrack-CMV at both ends (Table S1). The entire CDS of duck SOST was amplified by PCR. After sequencing, the amplified PCR products of SOST and PAdTrack-CMV plasmid, linearized with Xhol and Kpnl (Takara, China), were ligated E. coli DH5a homologous recombinase (Biodragon Immunotechnologies Co., Ltd, China). Then, the recombinant plasmids were confirmed by sequencing and digestion. The recombinant plasmids were named pAdTrack-CMV-duSOST. The pAdTrack-CMV-duSOST linearized with Pmel and the adenoviral backbone plasmid (pAdEasy-1) were ligated with homologous recombinant in E. coli BJ5183 (Miaoling Bioscience \& Technology Co., Ltd, China). The second recombinant plasmid was identified by Pacl (NEB, China) restriction enzyme digestion and sequenced, which showed that the vector was successfully constructed and named pAdduSOST (Fig. 1a). Finally, the second recombinant plasmid was subsequently used to generate recombinant adenoviruses in human embryonic kidney cells (HEK293; Kunming Institute of Zoology, China). The specific steps of adenovirus packaging in HEK293 cells are provided in the supplementary files.

\subsection{The titer determination of recombinant adenovirus}


The titer of adenovirus was measured according to Luo's article [14]. The HEK-293 cells were inoculated at $1 \times 10^{4}$ cells/well in a 96-well plate and $90 \mu \mathrm{L} /$ well complete culture medium containing $10 \%$ FBS after cell culture for 24 hours. Then, $10 \mu \mathrm{L}$ virus was added into the first well, and ten-fold dilution of the previous virus-containing solution was performed before the virus was added into the next well (12 wells in each group, altogether 3 groups) $\square$ After 48 hours, an inverted fluorescence microscope (OLYMPUS IX73, Japan) was used to observe the fluorescence-positive cells in each well[The virus titer was calculated according to the following equation; virus titer $(\mathrm{U} / \mathrm{mL})=\mathrm{m} \times 10^{\mathrm{n}+1}$, where $\mathrm{n}$ is the serial number of the reference well, and $\mathrm{m}$ is the number of positive cells!

\subsection{Duck myoblast isolation and culture}

The fertilizer eggs of ducks were collected from the Waterfowl Breeding Farm of Sichuan Agricultural University. The eggs were kept for incubating for 13 days under the same hatching conditions. According to the procedures of our previous studies, the isolation and cell culture of duck myoblasts were according to the guidelines of our previous studies [15]. The primary myoblasts of ducks were cultured in a complete growth medium containing DMEM/F-12 (HyClone, China), 10\% FBS, and 1\% penicillin/streptomycin, and then were incubated in an incubator with $5 \% \mathrm{CO}_{2}$ at $37^{\circ} \mathrm{C}$. All animal experiments were allowed by the Animal Ethics Committee of Sichuan Agricultural University.

\subsection{Multiplicity of infection (MOI) determination}

The duck primary myoblasts were inoculated at $1 \times 10^{5}$ cells/well in a 6-well plate, growing myoblasts (70\%-80\% confluent) were infected with adenovirus solution of $30,40,50,60$, and 70 multiplicity of infection (MOI), respectively. The cells were collected after 48 hours of infection, and the mRNA expression of the SOST gene in the cells was measured by real-time PCR.

\subsection{Transfection of pAd-duSOST to myoblasts}

The duck primary myoblasts were inoculated at $1 \times 10^{5}$ cells/well in a 6 -well plate and cultured in a complete medium (DMEM/F12 $+10 \% \mathrm{FBS}+100 \mathrm{U} / \mathrm{mL}$ penicillin and $100 \mathrm{~g} / \mathrm{mL}$ streptomycin). They were divided into the Ad-duSOST treatment group and the Ad-NC control group, with three replicates in each group. For proliferation assay, growing myoblasts (50\% confluent) were infected with optimal $\mathrm{MOI}$ of $\mathrm{Ad}-$ duSOST and Ad-NC. At 24 hours post-infected, cells were harvested for sampling. For differentiation assay, the culture medium was changed to differentiation medium (DM, DMEM/F12 containing $2 \%$ horse serum and $1 \%$ penicillin/ streptomycin) until approximately $80 \%$ confluence was reached, and the optimal $\mathrm{MOI}$ of Ad-duSOST and Ad-NC virus solution was added to each group. All cells were cultured in the incubator with $5 \% \mathrm{CO}_{2}$ at $37^{\circ} \mathrm{C}$. The cell samples infected by the virus were collected and stored at $-80{ }^{\circ} \mathrm{C}$ for RNA isolation.

\subsection{Cell proliferation assay}

Myoblasts viability was analyzed by a Cell Counting Kit-8 (CCK8, Bestbio Biotechnology, China). The primary myoblasts were seeded into a 96 -well plate with a density of $5 \times 10^{3}$ cells/well; after transfection 
Ad-duSOST or Ad-NC, myoblasts were incubated with $10 \mu \mathrm{L}$ CCK-8 for additional 3 hours at $37^{\circ} \mathrm{C}$; the absorbance at $450 \mathrm{~nm}$ was measured using a Microplate Reader (Thermo, USA). The samples from each treatment had six replicates at each time point.

Duck myoblasts were inoculated into 96-well plates with 2000-3000 cells per well with three repeats in each group. After 24 hours of treatment, the cells were incubated with EdU medium for 3 hours and then fixed with $4 \%$ paraformaldehyde. After that, the test was carried out according to the instructions of the EdU kit (Ribobio, China). Finally, it was observed and photographed under a fluorescence microscope.

\subsection{Flow cytometry assay}

Total $1 \times 10^{5}$ myoblasts were inoculated in a 6-well plate and transfected when the cell density was about 40-50\%. After 24 hours of transfection, the cells were digested with trypsin and collected. Then, the cells were washed once with PBS, centrifuged at $300 \mathrm{rpm}$ for $5 \mathrm{~min}$, and then the supernatant was discarded. The precipitation was fixed with ice-precooled $70 \%$ ethanol at $4^{\circ} \mathrm{C}$ for 18 hours. The samples were centrifuged at $300 \mathrm{rpm}$ for $5 \mathrm{~min}$, and the precipitate at the bottom was suspended with $1 \mathrm{~mL}$ PBS and filtered with a 300-mesh sieve. After centrifugation for $5 \mathrm{~min}$ at $300 \mathrm{rpm}$, PBS was discarded, and the precipitate was stained with $0.5 \mathrm{~mL}$ PI solution for $15 \mathrm{~min}\left(37^{\circ} \mathrm{C}\right.$, avoid light). The number of cells in each stage was analyzed by flow cytometry at $488 \mathrm{~nm}$ laser wavelength.

\subsection{Immunofluorescence}

Myoblasts were switched to differentiation medium (DM) for 2 days infected with Ad-duSOST or Ad-NC, and then these cells were used for immunofluorescence (IF) staining. The cells were fixed with $4 \%$ paraformaldehyde (PFA) for 30 min and treated with $0.1 \%$ Triton X-100 for 5 min at room temperature, then blocked with $5 \% \mathrm{BSA}$ for 1 hour. After incubation overnight at $4^{\circ} \mathrm{C}$ in a mouse primary polyclonal antibodies against MYHC (diluted 1:500; Biosynthesis Biotechnology, China), the cells were incubated against Cy3-conjugated secondary antibody (diluted 1:500; Beyotime Biotechnology, China). The slides were co-stained with 4, 6-diamidino-2-phenylindole (DAPI; Sigma) to visualize the nuclei. Then, the cells were visualized with an immunofluorescence microscope after having been washed in PBS thrice. The relative area of positive staining was evaluated with Image-Pro Plus 6.0 software (Media Cybernetics Corporation, USA).

\subsection{Western blot}

Total proteins were isolated by lysing cells in ice-cold RIPA lysis buffer. Protein samples from individual experiments were pooled for western blotting analysis. The primary antibodies were used; anti-MYHC (1:500 dilutions) and anti-GAPDH (1:1000 dilutions). The membranes were incubated with antibodies at $4^{\circ} \mathrm{C}$ overnight and washed in washing buffer $(10 \mathrm{mM}$ Tris- $\mathrm{HCl}, \mathrm{pH} 7.5,100 \mathrm{mM} \mathrm{NaCl}$, and $0.1 \%$ Tween 20). Next, the membranes were treated with horseradish peroxidase-conjugated IgG antibody (1:2000 dilutions) for 2 hours at room temperature. Each experiment was biologically replicated for triplicate times. Densitometry analysis of the bands relative to GAPDH was performed using Image $\mathrm{J}$ software (National Health Institute, Bethesda, MD, USA). 


\subsection{Real-time PCR}

Total RNA was extracted from myoblasts by RNAiso plus (Takara, Japan) according to the manufacturer's instructions and then measured by spectrophotometry. RNA was reverse-transcribed to synthesize the CDNA using the reverse transcript system (Takara, China). Real-time PCR was carried out with the SYBR Prime Script RT-PCR Kit (Takara, China) using the Bio-Rad CFX Manager (Bio-Rad Laboratories, USA). Expression of duck SOST, CDK6, PCNA, and CCND1/2 were detected, $\beta$-actin and GAPDH were used as inner controls. One sample collected from cells was repeated in triplicate. Primer sequences for real-time PCR are provided in Table S3. The relative expression levels of each gene were analyzed by the $2^{-\Delta \Delta C t}$ method[16].

\subsection{RNA-seq}

Two days' treatment after the myoblasts transfected with Ad-duSOST and Ad-NC, the cells were collected for sampling. Each treatment has three replicate samples. RNA was isolated, and its concentration and integrity were measured. High-quality RNA was sent to Novogene (Beijing, China) for cDNA libraries construction and sequencing. RNA-seq libraries were then generated. Briefly, after RNA fragmentation, double-stranded cDNA was synthesized by replacing dTTPs (deoxythymidine triphosphate) with dUTPs (deoxyuridine triphosphate) in reaction buffer used for second-strand cDNA synthesis. The resulting double-stranded CDNA was ligated to adaptors after being end-repaired and A-tailed. Single-strand cDNA was then obtained using the USER (Uracil-Specific Excision Reagent) Enzyme (NEB, Ipswich, U.K.). Finally, PCR amplification was performed to enrich the cDNA libraries. Sequencing was performed on an Illumina Hiseq 2500 instrument to generate 150-bp paired-end reads.

\subsection{Mapping and differentially expressed genes (DEGs) analysis}

Hisat2 (v2.1.0) built the index of genome files and mapped RNA-seq data to the reference genome (IASCAAS_PekingDuck_PBH1.5, GCF_003850225.1). StringTie (v1.3.3b) assembles the alignments into full and partial transcripts, and estimates the expression levels of all genes and transcripts, and creates read in files for Ballgown. Finally, the software packages in R (v3.5.1), such as gene filter, dplyr, and devtools, were used to analyze the differential genes. Functional groups and pathways encompassing the DEGs were identified based on GeneOntology (GO) and Kyoto Encyclopedia of Genes and Genomes (KEGG) pathway analysis using the KOBAS 3.0 software. The threshold was set as a $P \leq 0.05$.

\subsection{Statistical analysis}

The data were subjected to analysis of variance (ANOVA), and the means were compared for significance by Tukey's test using SPSS22 (IBM Corp, Armonk, NY). The results were expressed as the mean \pm SEM. A $p$-value of less than 0.05 was considered statistically significant.

\section{Results}




\subsection{Construction of duck SOST recombinant adenovirus vector}

The PCR amplification was carried out according to the primers listed in Table S1, and the PCR product of duck SOST with a length of $750 \mathrm{bp}$ was obtained (Fig. 1b). Then, the PCR product was constructed into pAdTrack-CMV, and the positive clones were identified by double enzyme digestion with $\mathrm{Xhol}$ and $\mathrm{Kpnl}$. Two fragments were obtained, about $750 \mathrm{bp}$ and $10 \mathrm{~kb}$, respectively (Fig. 1c). The sequencing works verified that the recombinant plasmid pAdTrack-CMV-duSOST has been constructed successfully, and afterward, they were co-transformed with pAdEasy-1 into BJ5183 (Fig. 1a). Later the recombinant plasmid was digested by Pacl, and obtained smaller and larger fragments with a length of $3 \mathrm{~kb}$ and 23.13 $\mathrm{kb}$, respectively (Fig. 1d). The sequence analysis verified that the recombinant adenovirus plasmid pAdduSOST was successfully constructed. Then, the pAd-duSOST was packaged in HEK293 cells to gain infection activities. After collecting the adenovirus, it was continuously amplified in HEK293 for three generations and found that the adenovirus had stable infection abilities and was named Ad-duSOST. Finally, the titer of recombinant adenovirus Ad-duSOST was $1 \times 108 \mathrm{U} / \mathrm{mL}$, tested by the LaSRT method (Fig.S1).

Duck myoblasts were infected by Ad-duSOST with $\mathrm{MOI}$ of 50, 60, 70, 80, and 90, respectively. After infection for 48 hours, the mRNA expression of SOST in myoblasts was measured by Real-time PCR (Fig.S2). The expression of SOST in myoblasts with different MOI was significantly higher than that of the Ad-NC group $(P<0.05)$. In addition, the mRNA expression of SOST in myoblasts with $80 \mathrm{MOI}$ was significantly higher than that in myoblasts with $70 \mathrm{MOI}(P<0.05)$.

\subsection{Effect of SOST on the proliferation of duck myoblasts}

To investigate the effects of SOST on myoblasts' proliferation, the AD-duSOST was transfected into duck primary myoblasts. Through the CCK-8 cell proliferation assay, it was observed that the cell proliferation activities in the treatment group were inhibited after 12,36, and 48 hours' treatment, respectively (Fig. 2a). The EdU can bind to single-strand DNA and specifically label the nuclei in the S phase of the cell cycle. The EdU assay showed that the nuclear labeled by Hoechst in myoblasts treated with Ad-duSOST was lower than that of the Ad-NC group. The labeling rates of EdU positive cells in the treatment group were significantly lower than the Ad-NC group $(P<0.05$, Fig. $2 b)$. In addition, flow cytometry showed that most myoblasts were blocked in the G0/G1 phase (Fig. 2C). There was no significant difference in the number of G0/G1 cells between the treatment group and the AD-NC group. The regulated factors of CDK6, PCNA, CCND1, and CCND2 were closely related to cell proliferation. Real-time PCR showed that all of them did not change under the treatments of Ad-duSOST (Fig. 2d).

\subsection{Effect of SOST on the differentiation of duck myoblasts}

The mRNA expression level of SOST in the myoblasts transfected with Ad-duSOST was significantly higher than that of the Ad-NC group. The SOST expressed to reach the maximum in myoblasts on the 
second day (D2) after transfection, whereas it gradually decreased on third (D3) and fourth (D4; Fig. 3a). Subsequently, immunofluorescence results showed that the fluorescence activity of MYHC decreased, and myotube formation was blocked by overexpressing SOST (Fig. 3b). MYHC is a marker gene of satellite cell differentiation, and its expression will significantly increase when the satellite cell begins to differentiate [17]. MYHC was detected by Western blot (Fig. 3c), and results showed that its protein level was decreased in myoblasts of the Ad-duSOST group than the Ad-NC group $(P>0.05)$.

\subsection{RNA-seq showed the main functional changes in myoblasts with SOST overexpression}

To further study the molecular changes in myoblasts during the differentiation process induced by overexpression of the SOST, the transcriptome profiling of myoblasts was compared between the AdduSOST infection group and the Ad-NC group. The details of RNA sequencing data reflecting the quality of sequencing works are provided in Table S4. The correlation analysis based on the TPM value of differentially expressed genes (DEGs) showed that the internal consistency of replicate samples in the same group was good (Fig. 4a). These results indicated that RNA sequencing data were of high quality with sufficient sequencing depth for the subsequent analysis.

A total of 331 DEGs were identified with significant foldchanges $(F C)$ of more than $1.8(P<0.05)$, including 196 and 134 were up-and down-regulated genes, respectively (Fig. 4b). Gene ontology (GO) terms showed that the DEGs were mainly enriched in development-related items, including regulating the multicellular organismal process, regulation of cell differentiation, and cell developmental process (Fig. 4c). The KEGG pathway enrichment showed that the DEGs were mainly enriched in muscle development (regulation of actin cytoskeleton), intercellular regulation, and disease-related (RAS signaling pathway) signaling pathway (Fig. 4d). Differential genes were also enriched in other pathways, such as Cytokine-cytokine receptor interaction and Wnt signal pathway, which involved cell growth, differentiation, and apoptosis [18]; [19] It is suggested that the SOST may affect the differentiation of duck myoblasts through these signal pathways.

\subsection{The genes involved in myoblast differentiation under the impacts of overexpression SOST}

To further understand the role of the SOST in myoblast, we found the top 20 DEGs $\left(P<0.05,\left|\log { }_{2} \mathrm{FC}\right|>3\right)$. Among them, ten genes were up-and down-regulated genes, respectively (Table S5). Through checking the functions of these twenty genes according to Genecards database (https://www.genecards.org/), it was found that these genes were mainly related to morphogenesis (NPS, BHLHA9, CLDN18, TAC1, ADA2, and FRK), disease (MYRFL, KLHDC7A, and NPY2R), and reproduction (ODF3, CRHBP, SPAM1, and DRC7). Among these genes, NPS has the highest Log2FC except MYRFL, which can promote myoblast differentiation [20]. Moreover, some genes related to myogenic differentiation (according to literature) were enriched (Table 1). Among them, BMP2 [21], SOX6, and TGFBR3 [22] were reported to inhibit the differentiation of myoblasts, while $\beta$-catenin (CTNNB1) was reported to promote the differentiation of 
myoblasts [23]. Moreover, BMP2, SOX6, and TGFBR3 were up-regulated, and CTNNB1 was downregulated gene. Afterward, the PPI analysis was performed based on these 14 genes and found direct interaction of the SOST with CTNNB1 and BMP2 (Fig. 5).

Table 1

The DEGs related to myoblast differentiation under the impacts of SOST overexpression

\begin{tabular}{|lll|}
\hline Genes & LOG2FC & P-value \\
\hline BMP2 & 1.17996 & 0.07269 \\
\hline BMPR1B & 0.76732 & 0.00262 \\
\hline GDF10 & 0.92765 & 0.04835 \\
\hline TGFBR3 & 1.12352 & 0.02167 \\
\hline ACVR1 & 0.99487 & 0.01038 \\
\hline FZD10 & 1.87914 & 0.00869 \\
\hline CTNNB1 & -2.21867 & 0.03428 \\
\hline GRIN2B & -1.12969 & 0.00587 \\
\hline ADORA1 & 1.26710 & 0.01187 \\
\hline MYOC & -1.07702 & 0.00208 \\
\hline SOX6 & 2.51936 & 0.01449 \\
\hline CBLN4 & -1.18442 & 0.47288 \\
\hline GATA4 & -1.20435 & 0.25342 \\
\hline INHBB & 0.75833 & 0.04666 \\
\hline
\end{tabular}

Note: TGFBR3 and CTNNB1 differed in transcripts.

\section{Discussion}

Both bone and muscle tissues are originated from mesenchymal stem cells in mesoderm during embryogenesis [1]. Studies on meat ducks have found that the sternum affects the development of corresponding pectoral muscles in the process of calcification. Moreover, bones and muscles are the largest endocrine organs in animals, synthesizing and secrete various cytokines to affect each other's development processes through paracrine pathways [4]. The SOST, a protein secreted by mature osteocytes, is a negative regulator of bone formation [24]. In duck, the higher expression of the SOST was found in the sternum, whereas lower expression was found in the pectoral muscle. Some reports suggested that the SOST may affect muscle development $[25,26,13]$. In this study, we observed the 
morphology of myoblasts at the cellular level. We found that SOST inhibited the differentiation of myoblasts, implying that the SOST may have multi-effect functions regulating the co-development of duck sternum and skeletal muscle.

Muscle development is a complex process, mainly including myoblast proliferation, myotube formation, and muscle fiber differentiation. In duck, we found that the SOST had an inhibition effect on myotube formation. Similarly, transcriptome revealed that the DEGs were mainly enriched in regulation of the multicellular organismal process, regulation of cell differentiation, and cell developmental process, as well as, six of the top 20 DEGs were related to morphogenesis. Studies showed that the SOST also has inhibition effects on osteoblast differentiation. When the SOST binds to the LRP5/ 6 and Frizzled coreceptors on the cell surface of osteoblasts, Wnt/ $\beta$-catenin signaling was inhibited [27]thereby inhibiting osteoblast differentiation $[28,29]$. The difference is that the SOST may inhibit osteoblast differentiation and promote apoptosis and inhibit cell proliferation in bone $[10,11]$. In vitro, the proliferation activity of MG63 cells was increased after silencing the expression of SOST [30]. In this study, we also verified the effect of SOST on the proliferation of myoblasts. However, flow cytometry results showed that the SOST overexpression did not inhibit cell cycle transition.

The mechanism of the SOST effect on the myoblasts differentiation is mainly related to the following pathways, such as Cytokine-cytokine receptor interaction, regulation of actin cytoskeleton, cAMP signaling pathway, and Wnt signaling pathway, which were reported to be involved in cell differentiation[31, 32, 19]We compiled a series of marker genes during myoblast differentiation, including myogenic regulatory factors (MRFs) family, myocyte enhancer factor 2 (MEF2) family, Pax3/7, and Myostatin (MSTN). The results showed that their P-value or Foldchanges were not within the range of DEGs. There are various reports indicated different DEGs and their role in cell differentiation. Up-regulated genes, such as BMP2, SOX6, and TGFBR3, were reported to inhibit the differentiation of myoblasts [18, $20,22,33]$. On the contrary, the down-regulated genes, such as $\beta$-catenin (CTNNB1), were reported to promote the differentiation of myoblasts [23]. Moreover, protein interaction network analysis showed that the SOST could directly interact with BMP2 and CTNNB1. Related studies also showed that the SOST could interact with BMP2 and CTNNB1 during osteoblast differentiation [34]. Therefore, we suspected that BMP2 and CTNNB1 might be involved in the process of SOST inhibiting myoblast differentiation.

Adenovirus vector is one of the most widely used viral vectors in the study of gene function, as its efficient gene transfer and protein expression in a variety of cells [35]. The shuttle plasmid contains a sequence encoding GFP, which is convenient for tracking and detection [36]. At present, there have been related studies to explore its role in osteoblasts by constructing adenovirus vectors that overexpress or silence SOST[30]. However, the possible role of SOST in the co-development of the duck sternum and pectoral muscle has not been reported. In the present study, we have constructed, for the first time, the adenovirus vector of duck SOST and observed its effect on duck myoblasts.

In the present study, the recombinant adenovirus vector expressing duck SOST was constructed successfully. We have confirmed that the formation of myotubes may be inhibited by SOST 
overexpression. Multiple genes and pathways were significantly involved in this process, including 14 genes such as CTNNB1 and BMP2 with a fundamental change of regulation modes. It is suggested that SOST may be a critical gene regulating the co-development of duck sternum and pectoral muscle.

\section{Declarations}

Funding $\triangle T$ This work was supported by the National Natural Science Foundation of China (31872345), Key Technology Support Program of Sichuan Province (2016NYZ0044), Agriculture Research System of China (CARS-43-6), and New breeding projects

Conflicts of interest/Competing interests[The authors declare that they have no conflicts of interest with the contents of this article.

Availability of data and material[The date that supports the findings of this study are available in the supplementary material of this article.

Authors' contributions $\square$ Hehe Liu and Yanying Li: Methodology, Visualization, Writing an original draft. Hehe Liu: Investigation, Methodology. Qian Xu: Visualization. Jianmei Wang: Visualization. Chunchun Han: Writing-review \& editing. Lili Bai: Writing-review \& editing. Liang Li: Writing-review \& editing

Consent to participate: All authors are acquainted with the manuscript

Consent for publication:Not applicable.

Ethics approval $\triangle$ The animal use protocol listed below has been reviewed and approved by the Sichaun Agricultural University Animal Ethical and Welfare Committee.

\section{References}

1. Hua L, Zhongjian X, Mei Z, Lin C (2016) Mutual regulation of muscle and bone. CHIN J OSTEOPOROSIS BONE MINER RES 9(3):236-239. 10.3969/j.issn.1674-2591.2016.03.003

2. Brotto M, Bonewald L (2015) Bone and muscle: Interactions beyond mechanical. Bone 80:109-114. 10.1016/j.bone.2015.02.010

3. Bonewald LF, Kiel DP, Clemens TL, Esser K, Orwoll ES, O'Keefe RJ, Fielding RA (2013) Forum on bone and skeletal muscle interactions: Summary of the proceedings of an ASBMR workshop. Journal of Bone \& Mineral Research the Official Journal of the American Society for Bone \& Mineral Research 28. $10.1002 /$ jbmr.1980

4. Karsenty G, Olson EN (2016) Bone and Muscle Endocrine Functions: Unexpected Paradigms of Interorgan Communication. Cell 164(6):1248-1256. https://doi.org/10.1016/j.cell.2016.02.043

5. Ken-Ichiro T, Erika M, Yoshiko H, Takenobu K, Toshitsugu S, Susumu S, Hiroshi K (2012) Role of osteoglycin in the linkage between muscle and bone. J Biol Chem 287(15):11616-11628. 
10.1074/jbc.M111.292193

6. Christian M, Roland K, Christina M, Arastoo Rahbar N, Katharina G, Heinrich M, Peter R, P (2015) Sclerostin levels and changes in bone metabolism after bariatric surgery. J Clin Endocr Metab 100(3):891-901. 10.1210/jc.2014-3367

7. Wosczyna MN, Biswas AA, Cogswell CA, Goldhamer DJ (2012) Multipotent progenitors resident in the skeletal muscle interstitium exhibit robust BMP-dependent osteogenic activity and mediate heterotopic ossification. Journal of Bone Mineral Research 27(5):1004-1017. 10.1002/jbmr.1562

8. Han Y, You X, Xing W, Zhang Z, Zou W (2018) Paracrine and endocrine actions of bone-the functions of secretory proteins from osteoblasts, osteocytes, and osteoclasts. Bone Res 6(2)):16.

10.1038/s41413-018-0019-6

9. Kim JH, Liu X, Wang J, Chen X, He TC (2013) Wnt signaling in bone formation and its therapeutic potential for bone diseases. Therapeutic Advances in Musculoskeletal Disease 5(1):13-31. $10.1177 / 1759720 \times 12466608$

10. Wijenayaka AR, Prideaux M, Yang D, Morris HA, Findlay DM, Anderson PH, Atkins GJ (2016) Early response of the human SOST gene to stimulation by 1alpha,25-dihydroxyvitamin D3. J Steroid Biochem Mol Biol 164:369-373. 10.1016/j.jsbmb.2015.12.006

11. Sutherland MK, Geoghegan JC, Yu C, Turcott E, Skonier JE, Winkler DG, Latham JA (2004) Sclerostin promotes the apoptosis of human osteoblastic cells: a novel regulation of bone formation. Bone 35(4):828-835. 10.1016/j.bone.2004.05.023

12. Spatz JM, Fields EE, Yu EW, Pajevic PD, Bouxsein ML, Sibonga JD, Zwart SR, Smith SM (2012) Serum Sclerostin Increases in Healthy Adult Men during Bed Rest. J Clin Endocr Metab 97 (9), E1736-E1740. 10.1210/jc.2012 - 1579

13. Huang J, Lara SR, Mo N, Kaja C, Brotto S, SI L, MI D, Jähn J, Lf K, B (2017) Crosstalk between MLO$\mathrm{Y} 4$ osteocytes and $\mathrm{C} 2 \mathrm{C} 12$ muscle cells is mediated by the Wnt/ $\beta$-catenin pathway. Jbmr Plus 1(2)):86. 10.1002/jbm4.10015

14. Wang ZQ (ed) (2011) Cloning, Sequence Analysis of CDS of PRLR and Construction of Adenovirus Vector for RNA Interference in Dairy Goat. Northwest A\&F University

15. Liu HH, Li L, Chen X, Cao W, Zhang RP, Yu HY, Xu F, He H, Wang JW (2011) Characterization of in vitro cultured myoblasts isolated from duck (Anas platyrhynchos) embryo. Cytotechnology 63(4):399406. https://doi.org/10.1007/s10616-011-9356-7

16. Livak KJ, Schmittgen TD (2001) Analysis of relative gene expression data using real-time quantitative PCR and the 2(-Delta Delta C(T)) Method. Methods 25(4):402-408. $10.1006 /$ meth.2001.1262

17. Charge P, SB (2004) Cellular and Molecular Regulation of Muscle Regeneration. Physiol Rev 84(1):209-238. 10.1152/physrev.00019.2003

18. Turner MD, Nedjai B, Hurst T, Pennington DJ (2014) Cytokines and chemokines: At the crossroads of cell signalling and inflammatory disease. Biochim Biophys Acta 1843(11):2563-2582.

10.1016/j.bbamcr.2014.05.014

Page 12/19 
19. Abu-Elmagd M, Robson L, Sweetman D, Hadley J, Francis-West P, Munsterberg A (2010) Wnt/Lef1 signaling acts via Pitx2 to regulate somite myogenesis. Dev Biol 337(2):211-219.

10.1016/j.ydbio.2009.10.023

20. Lambert O, Poussard S, Decossas M, Le Bihan O, Mornet S, Naudin G, Internalization and fate of silica nanoparticles in $\mathrm{C} 2 \mathrm{C} 12$ skeletal muscle cells: evidence of a beneficial effect on myoblast fusion. Int. J. Nanomed. 10.2147/IJN.S74158

21. Droguett R, Cabello-Verrugio C, Santander C, Brandan E (2010) TGF- $\beta$ receptors, in a Smadindependent manner, are required for terminal skeletal muscle differentiation. 316 (15), 2487-2503. 10.1016/j.yexcr.2010.04.031

22. Droguett R, Cabello-Verrugio C, Riquelme C, Brandan E (2006) Extracellular proteoglycans modify TGF-beta bio-availability attenuating its signaling during skeletal muscle differentiation. Matrix Biol 25(6):332-341. 10.1016/j.matbio.2006.04.004

23. Brack AS, Conboy IM, Conboy MJ, Shen J, Rando TA (2008) A Temporal Switch from Notch to Wnt Signaling in Muscle Stem Cells Is Necessary for Normal Adult Myogenesis. 2 (1), 0-59.

10.1016/j.stem.2007.10.006

24. Compton JT, Lee FY (2014a) A Review of Osteocyte Function and the Emerging Importance of Sclerostin. Journal of Bone Joint Surgery American Volume 96(19):1659-1668

25. Hesse E, Schroder S, Brandt D, Pamperin J, Saito H, Taipaleenmaki H (2019) Sclerostin inhibition alleviates breast cancer-induced bone metastases and muscle weakness. JCI Insight 5. 10.1172/jci.insight. 125543

26. Wood CL, Pajevic PD, Gooi JH (2017) Osteocyte secreted factors inhibit skeletal muscle differentiation. Bone Reports 6:74-80. 10.1016/j.bonr.2017.02.007

27. Li X, Zhang Y, Kang H, Liu W, Liu P, Zhang J, Harris SE, Wu D (2005) Sclerostin binds to LRP5/6 and antagonizes canonical Wnt signaling. J Biol Chem 280(20):19883-19887.

10.1074/jbc.M413274200

28. Baron R, Rawadi G (2007) Targeting the Wnt/beta-catenin pathway to regulate bone formation in the adult skeleton. Endocrinology 148 (6), 2635-2643. 10.1210/en.2007 - 0270

29. Compton JT, Lee FY (2014b) A Review of Osteocyte Function and the Emerging Importance of Sclerostin. Journal of Bone Joint Surgery American Volume 96(19):1659-1668. 10.2106/JBJS.M.01096

30. Lei W, Hongxing $\mathrm{H}$, Hong $\mathrm{H}$ (2016) Construction of DKK1 and Sost recombinant adenovirus silencing vector and their effect on MG63 cell viability, ALP activity and Ca2 + concentration. Chinese Journal of Osteoporosis 22(11):1361-1369. 10.3969/j.issn.1006-7108.2016.11.001

31. Tang DD, Anfinogenova Y (2008) Physiologic Properties and Regulation of the Actin Cytoskeleton in Vascular Smooth Muscle. Journal of Cardiovascular Pharmacology Therapeutics 13(2):130-140. $10.1177 / 1074248407313737$

32. Sruti C, Jolyon T, Zhang G, Sriharsa P, Stephen H, Douglas J, Carl B, Michelle L, Melanie E (2015) Tissue-specific epigenetics in gene neighborhoods: myogenic transcription factor genes. Hum Mol 
Genet (16), 16. 10.1093/hmg/ddv198

33. Erika S, Takeo S, Akira Y, Kayoko A, Tadayoshi F, Yoshiki N (2011) Bone morphogenetic protein-2 functions as a negative regulator in the differentiation of myoblasts, but not as an inducer for the formations of cartilage and bone in mouse embryonic tongue. BMC Dev Biol 11(1):44. 10.1186/1471-213X-11-44

34. Zhang Y, Yao-Hua HE (2015) Effect of Sclerostin on BMP-2-induced osteogenesis of human mesenchymal stem cells. Shandong Medical Journal. 10.3969/j.issn.1002-266X.2015.28.002

35. Wirth T, Ylä-Herttuala S (2014) Gene transfer vectors (DNA vehicles) and their incorporation into biomaterials for bone repair. Biomaterials for Bone Regeneration. 10.1533/9780857098104\$43.374

36. Li X, Liu Q, Bi X, Sheng G, Fu J (2008) An in vitro model to evaluate virus aerosol characteristics using a GFP-expressing adenovirus. J Med Microbiol 57(Pt 11):1335-1339.

10.1099/jmm.0.2008/000612-0

\section{Figures}


a

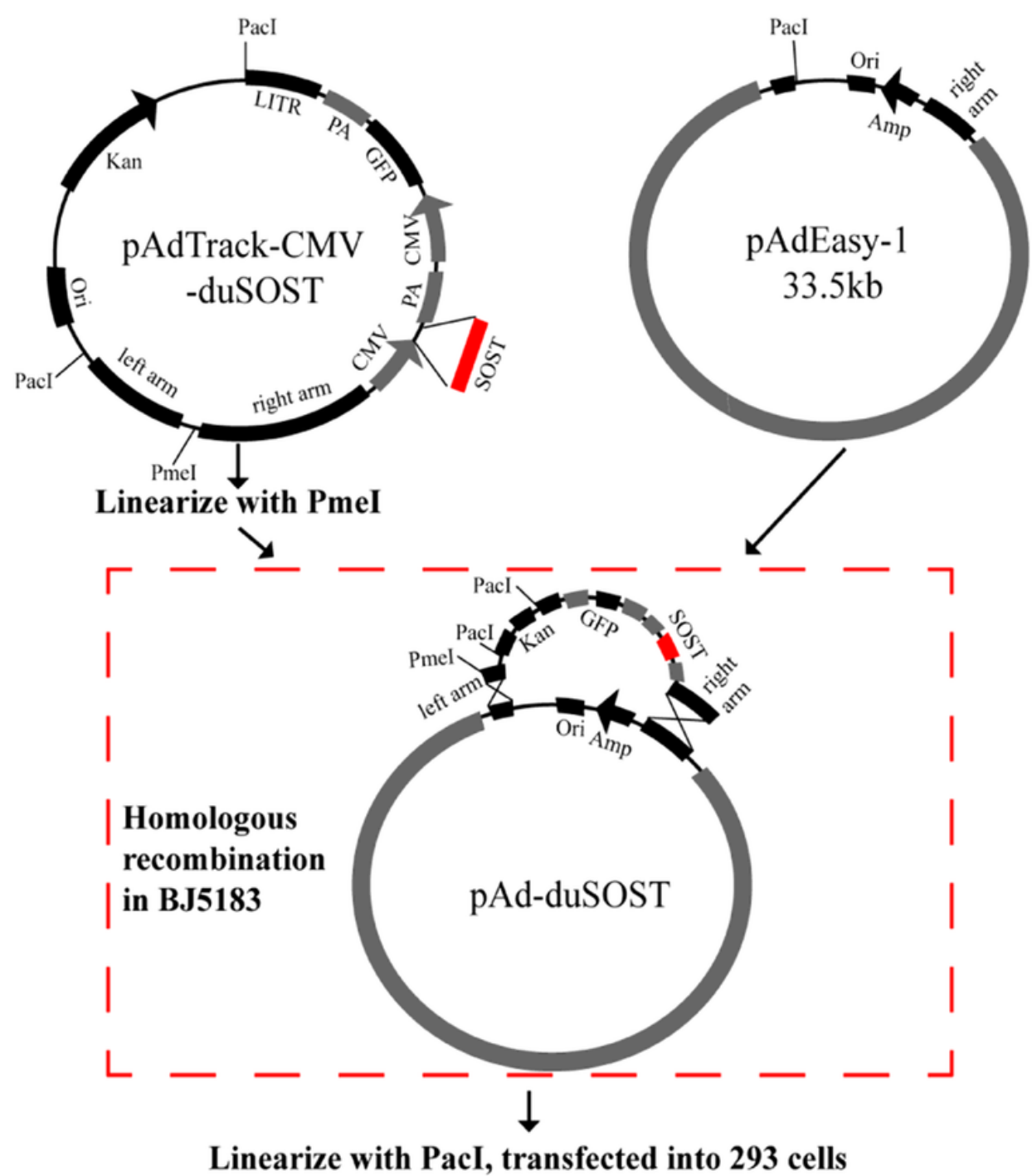

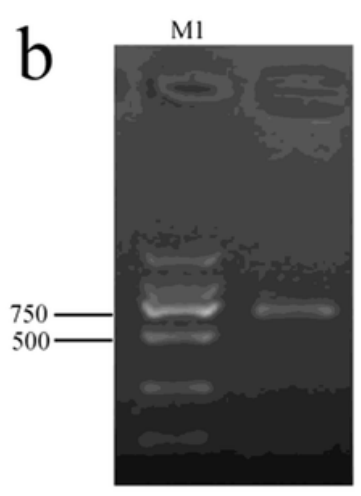

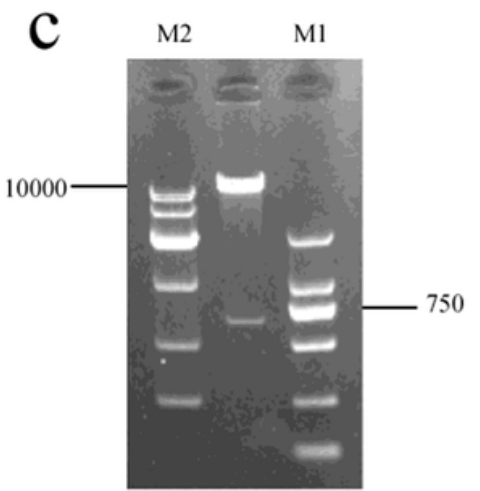

d

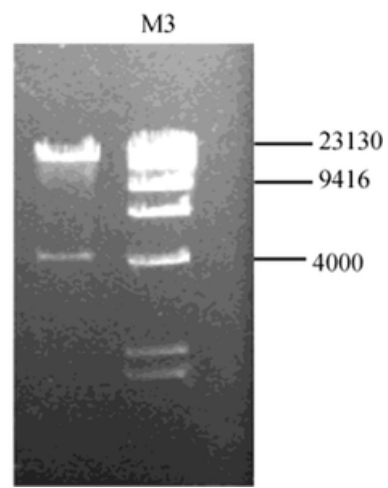

Figure 1

Construction and identification of recombinant adenovirus vector expressing duck SOST a: A schematic route to show recombinant adenovirus vector expressing duck SOST by AdEasy adenovirus system; $b$ : PCR products of SOST coding region. c: Identification of PAdTrack-CMV-duSOST plasmid by double enzyme digestion reactions (Xhol and Kpnl). d: Identification of pAd-duSOST plasmid by Pacl enzyme digestion. M1-M2 is a 2000 and 10000 bp DNA Marker. M3 is $\lambda$-Hind III digest. 
a

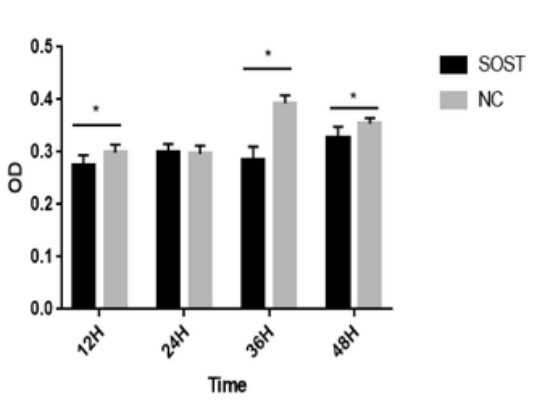

b
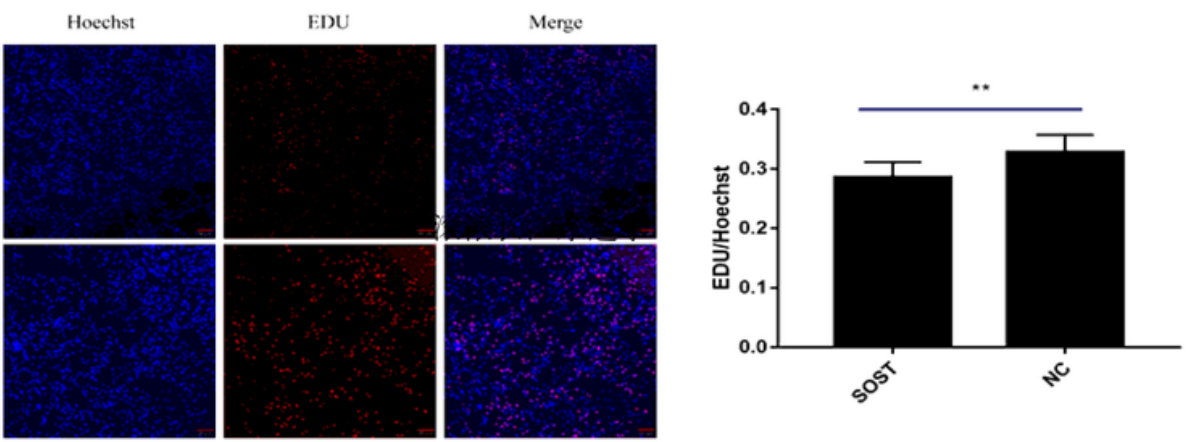

d
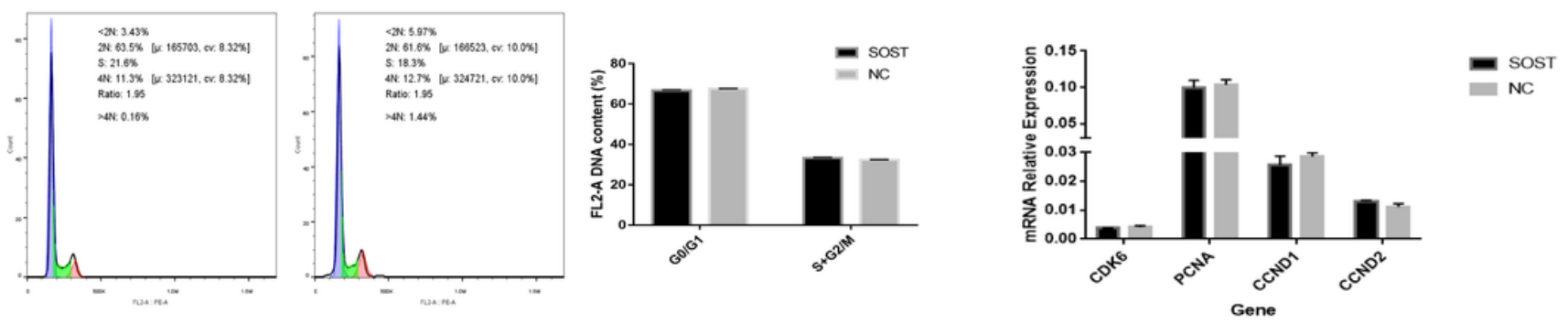

Figure 2

Effects of overexpression SOST on myoblasts' proliferation states of ducks. a: The proliferation rate of myoblasts was detected by CCK-8 at different time points. The amount of form-azan produced is directly proportional to the number of living cells and can be meas-ured by the absorbance at $450 \mathrm{~nm}$. b:

Schematic diagram of myoblasts labeled by EdU. All nucleus labeled by Hoechst is marked in blue, while only proliferative and mitotic nuclei are embedded by EdU and marked in red. c: The cell cycle changes of duck my-oblasts were determined by flow cytometry. $\mathrm{d}$ : The mRNA expression levels of marker genes related to cell proliferation. 
a

C

NC SOST

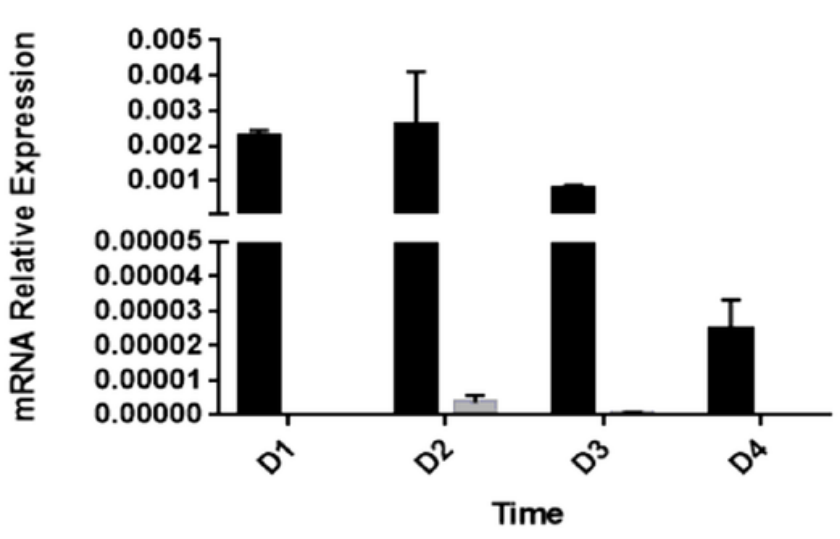

GAPDH

SOST

NC

MYHC

b

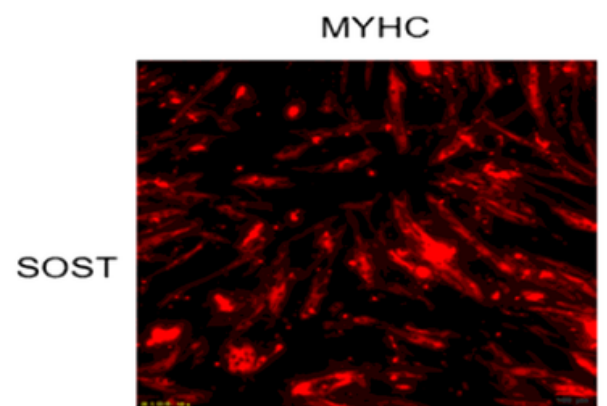

DAPI
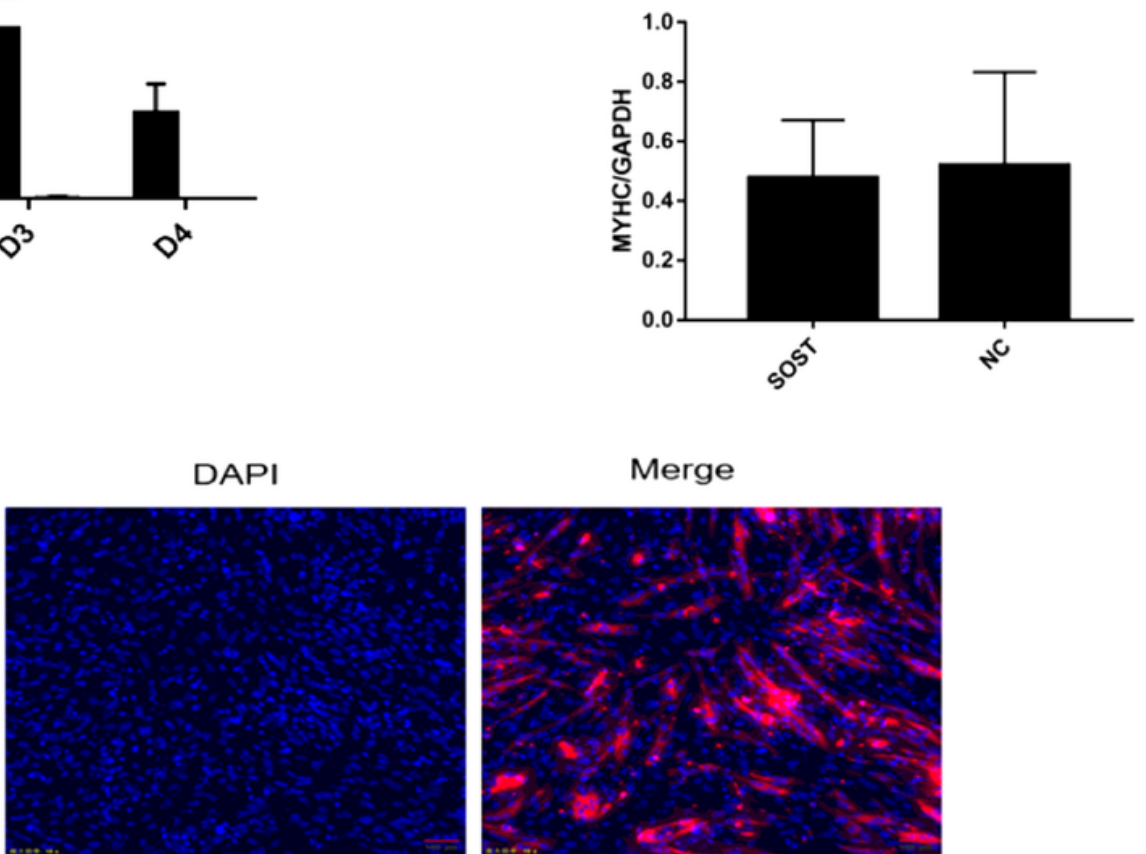

NC
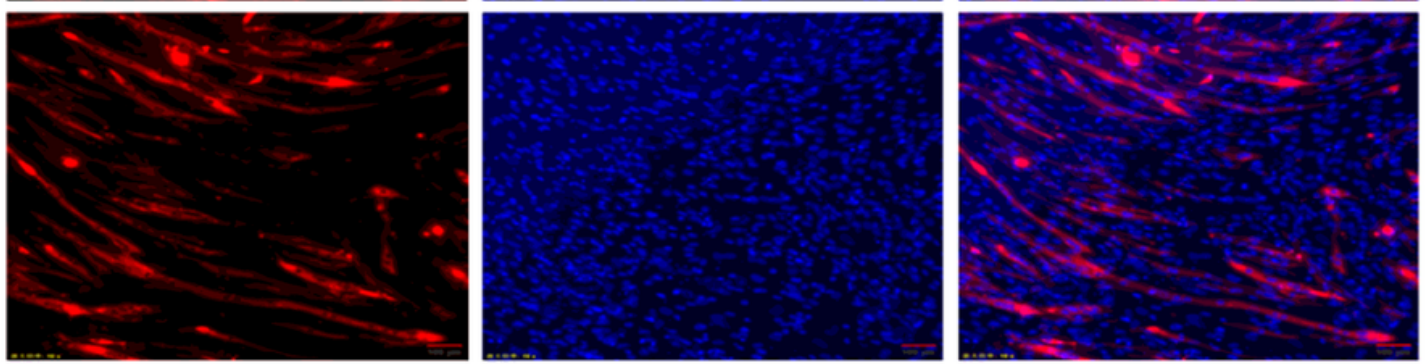

Figure 3

Effects of overexpression SOST on myoblasts' differentiation a: The mRNA expression pro-files of SOST in myoblasts after transfected with Ad-duSOST. b: The immunofluores-cence was used to detect the MYHC in the myoblast. The scale bar is $50 \mu \mathrm{m}$. c: The protein content of MYHC, detected by Western Blot, and GAPDH was used as the ref-erence protein. 
a

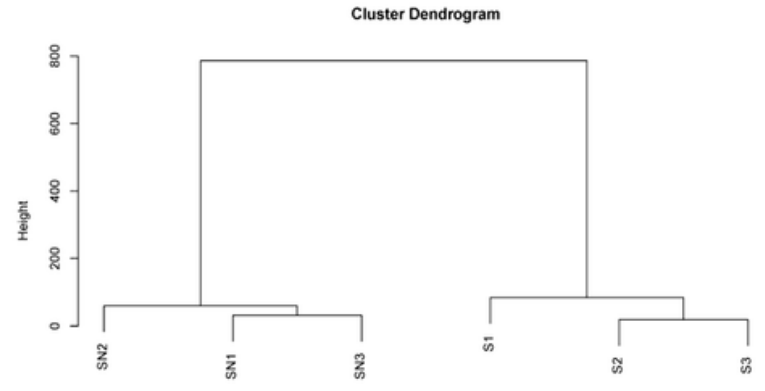

c
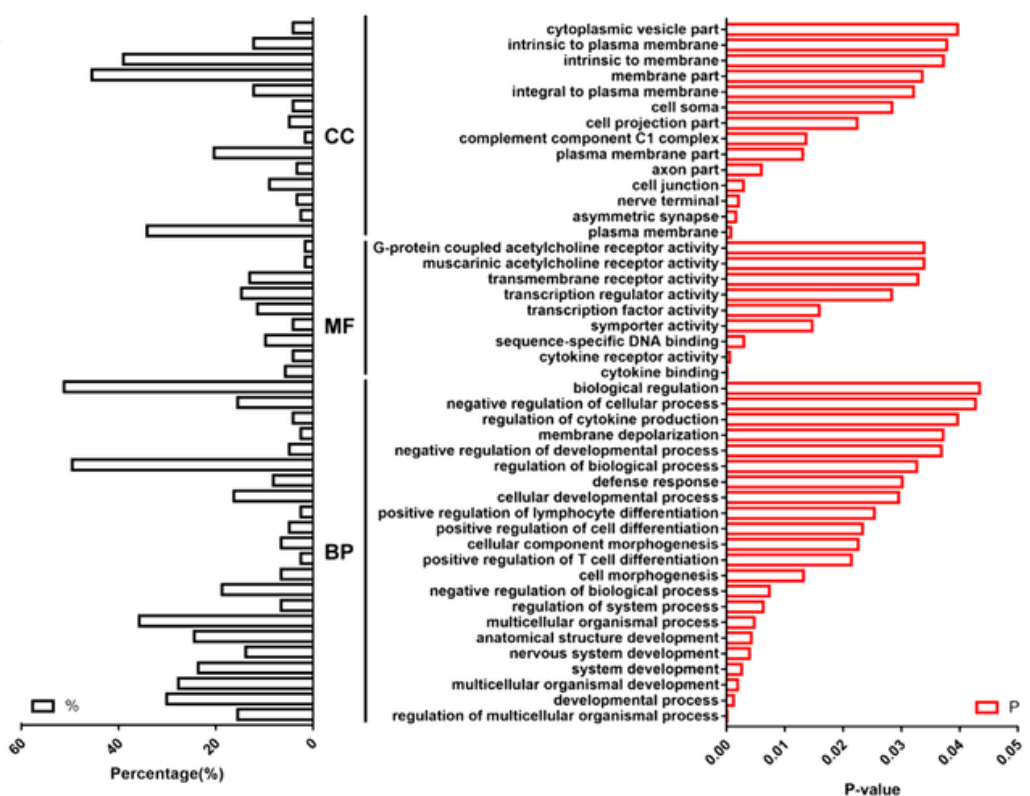

b

UP, DOWN regulated count

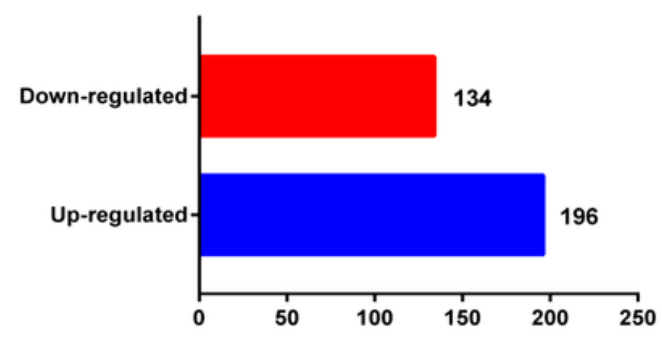

d

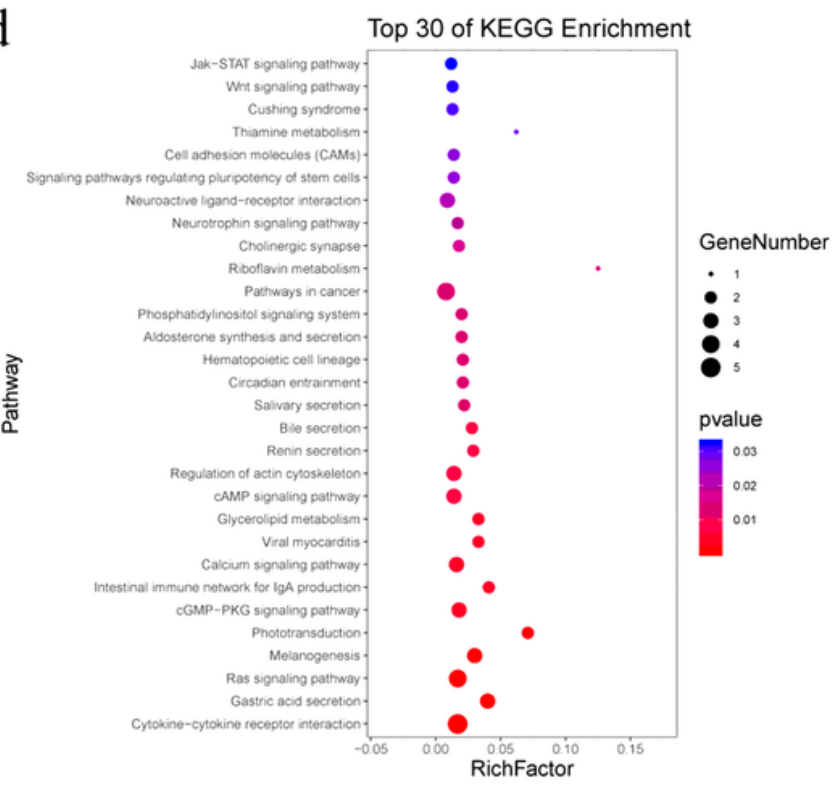

\section{Figure 4}

Comparison of transcriptome between Ad-duSOST and Ad-NC group a: Sample correlations based on genome-wide gene expression of each sample. b: Statistics of the number of differential expressed genes (DEGs). c: The GO analysis is based on the DEGs. d: The KEGG pathway analysis based on the DEGs. 


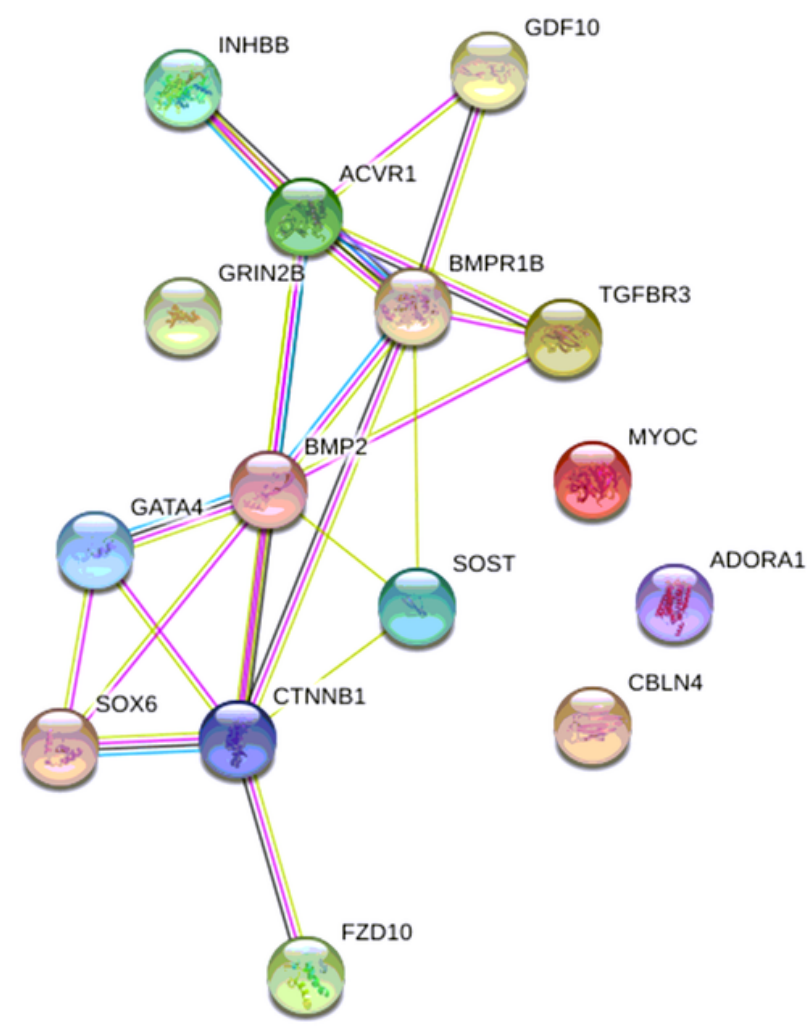

Figure 5

PPI networks between SOST and 14 DEGs related to cell differentiation The PPI was performed in the STRING database.

\section{Supplementary Files}

This is a list of supplementary files associated with this preprint. Click to download.

- Supplementaryfile.docx 